\title{
Structural and Optical Properties of the Perovskite Layer on Well-Aligned ZnO Nanorods
}

Received July 15, 2020; accepted July 23, 2020

\author{
Jaewon Oh and Mee-Yi Ryu* \\ Department of Physics, Kangwon National University, Gangwon-do 24341, Republic of Korea
}

*Corresponding author E-mail: myryu@kangwon.ac.kr

\begin{abstract}
To achieve highly efficient perovskite solar cells, it is important to design a metal oxide layer with high electron mobility. In this study, well-aligned $\mathrm{ZnO}$ nanorods (NRs) were prepared through the hydrothermal method using $\mathrm{ZnO}$ seed layers with varying thickness. The effect of the structural and optical properties of the perovskite layer deposited under the prepared conditions was systematically investigated via scanning electron microscopy, X-ray diffraction, ultraviolet-visible spectroscopy, and photoluminescence (PL) spectroscopy. The improved alignment of the $\mathrm{ZnO} \mathrm{NRs}$ was found to affect the topography of the perovskite polycrystals, such as increased grain size and improved uniformity of the perovskite layer. The thicker the $\mathrm{ZnO}$ seed layer, the more enhanced the alignment of the $\mathrm{ZnO} \mathrm{NRs}$, resulting in larger and more uniform grains. The perovskite layer deposited on the well-aligned $\mathrm{ZnO}$ NRs exhibited increased absorbance and PL intensity without changing crystallinity. Our results will help improve the efficiency of high-quality perovskite devices based on $\mathrm{ZnO}$ electron transport layer.
\end{abstract}

Keywords: Perovskite, $\mathrm{ZnO}$ nanorod, Electron transport layer, Photoluminescence, Scanning electron microscope

\section{Introduction}

The tremendous advancement in perovskite solar cell (PSC) devices began with the use of an absorber layer in dye-sensitized solar cells. In this study, mesoporous $\mathrm{TiO}_{2}$ was used to increase the contact area with perovskite and efficiently extract photogenerated electrons [1]. However, although the interface between the perovskite and the electron transport layer (ETL) inner surface can sometimes be an important factor in the performance of the solar cell, this interface cannot be easily controlled due to various complexities [2]. In a planar PSC architecture, the ETL is usually located directly at the bottom before the perovskite absorber is deposited. Thus, not only the topography and structure of the ETL surface greatly affect the interface, but also perovskites can affect the formation of the film. In addition, to achieve highly efficient PSCs, a metal oxide layer with high electron mobility should be designed [3]. Recently, many stable and efficient metal oxide materials used in PSCs, such as $\mathrm{TiO}_{2}, \mathrm{ZnO}$, and $\mathrm{SnO}_{2}$, have been reported [4-6]. Among them, $\mathrm{TiO}_{2}$ has been widely used as a suitable ETL. However, $\mathrm{TiO}_{2}$ requires a high annealing temperature above $\sim 500{ }^{\circ} \mathrm{C}$, which will be an obstacle in the development of PSCs in future flexible substrates. $\mathrm{ZnO}$ has a similar bandgap and physical properties with $\mathrm{TiO}_{2}$ and can be synthesized at a lower temperature than $\mathrm{TiO}_{2}$, making it a suitable alternative for high-efficiency PSCs [7]. Nie et al. reported that the morphological factors of perovskite grains include the performance of PSCs and stability [8]. Thus, a deep understanding on the formation of the perovskite layer with the applied ETL is valuable in creating stable and highly efficient PSCs.

In this study, well-aligned $\mathrm{ZnO}$ nanorods (NRs) were synthesized by the hydrothermal method by increasing the thickness of the $\mathrm{ZnO}$ seed layer through the sol-gel method to dramatically change the morphology of the ETL surface. Then, the perovskite layer was deposited by a one-step anti-solvent quenching method. The improved alignment of the $\mathrm{ZnO}$ NRs greatly influenced the formation of perovskite polycrystals. We subsequently investigated the structural and optical properties of the perovskite layer by using scanning electron microscopy (SEM), X-ray diffraction (XRD), ultraviolet-visible (UV-vis) absorbance, and photoluminescence (PL) spectroscopy.

\section{Experimental details}

ZnO NRs were synthesized on fluorine-doped tin oxide (FTO) glass substrates by a two-step process. First, a $\mathrm{ZnO}$ seed layer was deposited on the substrates using a sol-gel method, and then $\mathrm{ZnO}$ NRs were formed on the seed layer by the hydrothermal method. All materials used for the $\mathrm{ZnO}$ synthesis were purchased and used immediately without further purification. The $\mathrm{ZnO}$ seed precursor solution $(0.5 \mathrm{M})$ was prepared by dissolving zinc acetate dihydrate (Alfa Aesar) in 2-methoxyethanol (Alfa Aesar), and ethanolamine (Alfa Aesar) was added as a stabilizer to form a mixed solution. The mixture was stirred for $60 \mathrm{~min}$ at $60{ }^{\circ} \mathrm{C}$ on a hot plate to obtain a transparent and homogeneous solution. The resultant sol was aged for 24 hours at room temperature. The sol solution self-reacted through aging and was spin-coated on the FTO substrate for $30 \mathrm{~s}$ at a rotation speed of $3000 \mathrm{rpm}$ under ambient conditions. The resultant seed layer was then dried on the hot plate for $10 \mathrm{~min}$ at $300{ }^{\circ} \mathrm{C}$. To obtain different thicknesses of the $\mathrm{ZnO}$ seed layer, the spin-coating and drying processes were repeated for 1 to 10 times. ZnO NRs were 
formed on the $\mathrm{ZnO}$ seed layer in the hydrothermal solution. The hydrothermal solution was prepared by mixing $0.05 \mathrm{M}$ zinc nitrate hexahydrate (Alfa Aesar) and equimolar ratio hexamethylenetetramine (Alfa Aesar) in deionized water (Sigma-Aldrich). The aqueous solution was stirred for $60 \mathrm{~min}$ at room temperature. In the beaker with the hydrothermal solution, the $\mathrm{ZnO}$ seed layer face was placed at an angle toward the bottom face of the beaker to prevent precipitation of residues and was sealed. To perform a hydrothermal reaction, the beaker was placed in an oven at a constant temperature for $60 \mathrm{~min}$ at $95{ }^{\circ} \mathrm{C}$. After the hydrothermal reaction was completed, the beaker was cooled in an ambient environment, rinsed several times with deionized water, and then dried on a hot plate for $10 \mathrm{~min}$ at $150{ }^{\circ} \mathrm{C}$.

For the synthesis of the $\mathrm{CH}_{3} \mathrm{NH}_{3} \mathrm{PbI}_{3}$ perovskite precursor solution, the recipe previously reported by our group was used [9]. The grown $\mathrm{ZnO}$ NR layer was exposed to the UV-ozone for $15 \mathrm{~min}$ at $100{ }^{\circ} \mathrm{C}$ before depositing the perovskite layer, and then an anti-solvent quenching method was applied to deposit the $\mathrm{CH}_{3} \mathrm{NH}_{3} \mathrm{PbI}_{3}$ solution and annealed for $1 \mathrm{~min}$ at $100{ }^{\circ} \mathrm{C}$.

XRD measurements were conducted using X'pert Pro X-ray diffractometer (Malvern Panalytical). An ultra-high-resolution SEM (S-4800, Hitachi) was used to examine the morphology of the layer. Absorbance was measured using a Libra S80 spectrometer (Biochrom Ltd.). The PL measurement was performed using a silicon photodiode (Electro-Optical Systems Inc.) with a diode pump solid state laser $(\lambda=$ $532 \mathrm{~nm}$; Changchun New Industries Optoelectronics Technology Co., Ltd.) as the excitation source.

\section{Results and discussion}

Figure 1 shows the ultra-high-resolution SEM surface images of the $\mathrm{ZnO}$ seed layer, $\mathrm{ZnO} \mathrm{NRs} / \mathrm{ZnO}$ seed layer, and perovskite/ZnO $\mathrm{NRs/ZnO}$ seed layer, with S\#, NS\#, and PNS\# as the number of the $\mathrm{ZnO}$ seed layer deposition repetition times, respectively. As the number of deposition repetition times of the $\mathrm{ZnO}$ seed layer increased from 1 to 10, the thickness of the seed layer became thicker from $\sim 50$ to $\sim 200 \mathrm{~nm}$, and the rough texture of the FTO substrate surface gradually mitigated. Finally, as shown in Figs. 1(a) and 1(d), the ZnO seed layer completely covered the rough surface of the FTO substrate. Generally, ZnO NRs grown by the hydrothermal method prefer to grow in a direction perpendicular to the substrate surface [10]. The NRs with a thin seed layer mostly grew at an angle [Fig. 1(b)], whereas the NRs with a thick seed layer grew perpendicular to the plane [Fig.

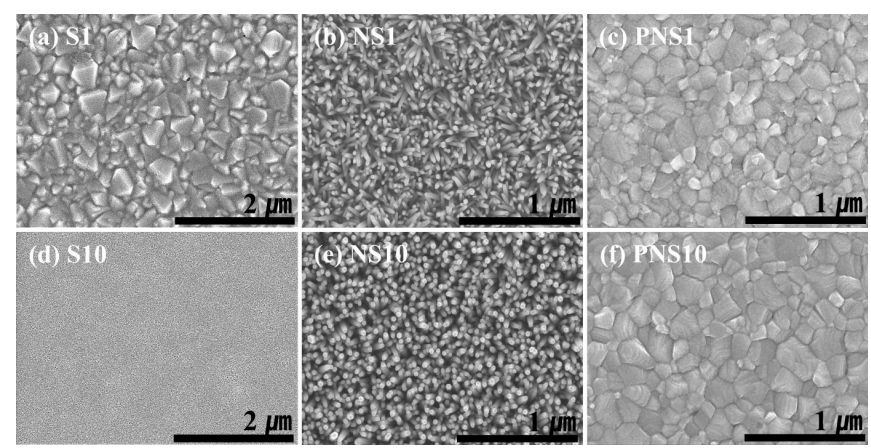

Figure 1. Surface scanning electron microscope images of ( $a$ and $d)$ the $\mathrm{ZnO}$ seeds, ( $b$ and e) $\mathrm{ZnO}$ nanorods, and ( $\mathrm{c}$ and f) perovskite samples with varying $\mathrm{ZnO}$ seed layer thickness. 1(e)]. Hence, the $\mathrm{ZnO}$ NRs grown on the $\mathrm{ZnO}$ seed layer with improved surface texture (NS10) matured as well-aligned NRs due to the flat bottom surface. All the grown NR samples were synthesized under the same hydrothermal conditions (precursor concentration, growth temperature, and time), so they had similar diameters $(\sim 30$ $\mathrm{nm})$. However, due to the difference in alignment, the overall thickness of the NR layers was thicker as the alignment was improved. The influence of the perovskite layer formation on the alignment of the NRs is clearly shown in Figs. 1(c) and 1(f). The surface of the PNS1 sample showed the formation of rough, small, and irregular grains, whereas the PNS10 sample had a larger and more uniform grain and a more compact surface.

Figure 2 shows the XRD spectra of the $\mathrm{CH}_{3} \mathrm{NH}_{3} \mathrm{PbI}_{3}$ samples deposited on $\mathrm{ZnO}$ NRs measured at different diffraction angles $\left(10^{\circ}\right.$ to $\left.50^{\circ}\right)$. Relatively strong peaks appear in all samples at $34.45^{\circ}$, which is the (002) diffraction peak of the $\mathrm{ZnO}$ NRs. Hence, the $\mathrm{ZnO}$ NRs grown by the hydrothermal method preferred to grow in the (002) direction. As the number of the $\mathrm{ZnO}$ seed layer deposition repetition times increased, the intensity of the (002) peak increased because the orientation of the $\mathrm{ZnO}$ NRs was improved and was in good agreement with the SEM image results. Zhang and Pauporté [11] reported that depositing a perovskite on a $\mathrm{ZnO}$ material using a one-step solution process easily caused deterioration at the perovskite/ $\mathrm{ZnO}$ interface during the annealing process, lowering the film quality compared with that in the two-step process. However, no residual $\mathrm{PbI}_{2}$ diffraction peak around $12.7^{\circ}$ was observed in all our prepared samples. In addition, strong (110) peaks and other (220) and (310) diffraction peaks indicate that the precursor solution was well converted to the perovskite phase without degradation. The major perovskite (110) diffraction peaks of the three samples at $14.15^{\circ}$ showed a similar intensity. This finding suggests that improving the orientation of the $\mathrm{ZnO}$ NRs changes the surface morphology without affecting the crystallinity of the perovskite layer. Consequently, we concluded that the improvement of the orientation of the $\mathrm{ZnO}$ NRs changed the grain size and uniformity without affecting the crystallinity of the perovskite.

We measured the UV-vis absorbance and PL to investigate the optical properties of the perovskite layer on well-aligned $\mathrm{ZnO}$ NRs. In Fig. 3(a), the onset of the absorption of all samples was measured near $783 \mathrm{~nm}$. The thicker the $\mathrm{ZnO}$ seed layer (PNS10), the better the

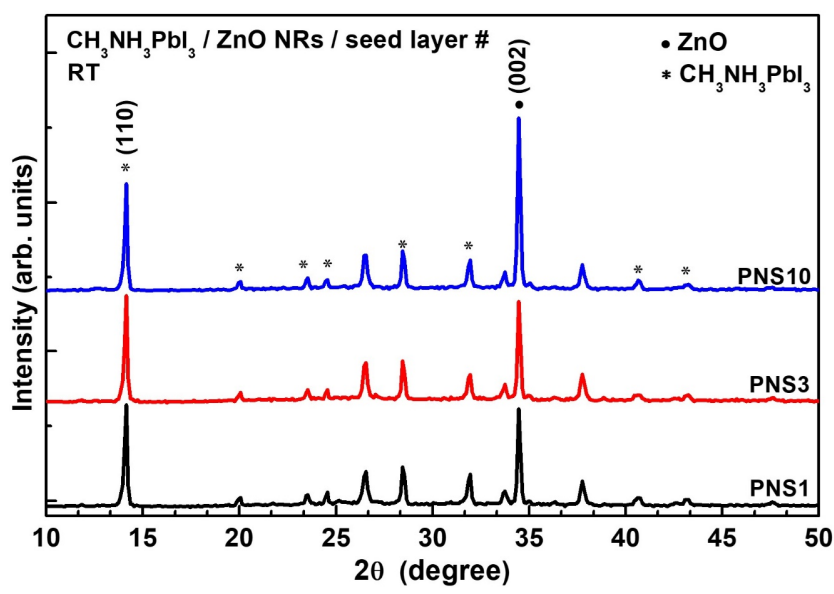

Figure 2. X-ray diffraction patterns of the perovskite layer with different $\mathrm{ZnO}$ nanorod alignments. 

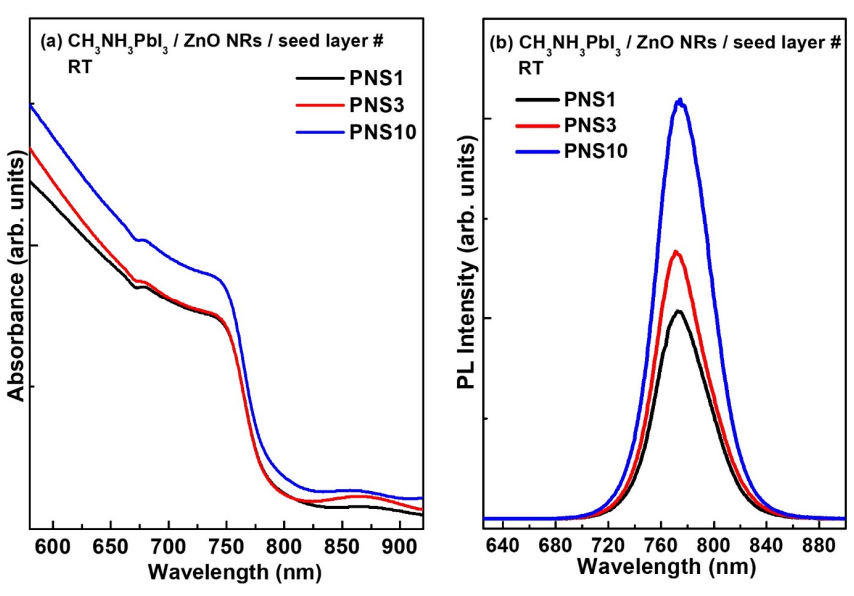

Figure 3. Room-temperature (a) UV-vis absorbance and (b) PL spectra of the perovskite layer.

absorbance over the entire wavelength range. This result is related to the large grain size and increased uniformity in the SEM image of Fig. 1(f). Wen et al. reported an increase in absorbance to enhanced grain size and uniformity [12]. The intensity of the PL spectra in Fig. 3(b) gradually increased as the thickness of the seed layer increased. Nukunudompanich et al. adjusted the perovskite grains to improve the performance of the device [13]. Herein, ETL changed the perovskite grain size and uniformity by obtaining different surface roughness by applying different $\mathrm{TiO}_{2}$ synthesis methods. Larger grains were obtained on small roughness surfaces. In our case, well-aligned NRs (NS10) would have a smaller surface roughness compared with samples with a relatively unaligned thin seed layer (NS1), which would affect the formation of perovskite polycrystals, such as less void formation at the interface between perovskite and $\mathrm{ZnO}$ NRs. Therefore, as the alignment of the NRs improves, the size of the perovskite grain becomes larger and more uniform. Clearly, the $\mathrm{CH}_{3} \mathrm{NH}_{3} \mathrm{PbI}_{3}$ film grown on the well-aligned $\mathrm{ZnO} \mathrm{NR}$ layer demonstrated improved morphology with a large grain size and good uniformity, resulting in enhanced absorbance and PL intensity.

\section{Conclusions}

We investigated the structural and optical properties of the perovskite layer on well-aligned $\mathrm{ZnO}$ NRs by adjusting the thickness of the $\mathrm{ZnO}$ seed layer with changing the number of deposition repetition times. The $\mathrm{ZnO}$ seed layer completely covering the surface of the FTO substrate improved the orientation of the ZnO NRs, thereby increasing the grain size and uniformity of the perovskite layer. This increased grain size and uniformity enhanced the optical properties of the perovskite layer by reducing grain boundaries. Our results will help improve the efficiency of high-quality perovskite devices based on ZnO ETLs.

\section{Acknowledgements}

This research was supported by the Basic Science Research Program through the National Research Foundation of Korea (NRF) funded by the Ministry of Education, Science, and Technology (NRF-2019R1A2C1086813).

\section{References}

[1] A. Kojima, K. Teshima, Y. Shirai, and T. Miyasaka, J. Am. Chem. Soc. 131, 6050 (2009).

[2] K. Schutt, P. K. Nayak, A. J. Ramadan, B. Wenger, Y. H. Lin, and H. J. Snaith, Adv. Funct. Mater. 29, 1 (2019).

[3] J. P. Correa-Baena, A. Abate, M. Saliba, W. Tress, T. J. Jacobsson, M. Grätzel, and A. Hagfeldt, Energy Environ. Sci. 10, 710 (2017).

[4] J. Burschka, N. Pellet, S. J. Moon, R. Humphry-Baker, P. Gao, M. K. Nazeeruddin, and M. Grätzel, Nature 499, 316 (2013).

[5] J. Dong, Y. Zhao, J. Shi, H. Wei, J. Xiao, X. Xu, J. Luo, J. Xu, D. Li, Y. Luo, and Q. Meng, Chem. Commun. 50, 13381 (2014).

[6] W. Ke, G. Fang, Q. Liu, L. Xiong, P. Qin, H. Tao, J. Wang, H. Lei, B. Li, J. Wan, G. Yang, and Y. Yan, J. Am. Chem. Soc. 137, 6730 (2015).

[7] J. Luo, Y. Wang, and Q. Zhang, Sol. Energy 163, 289 (2018).

[8] W. Nie, H. Tsai, R. Asadpour, J. C. Blancon, A. J. Neukirch, G. Gupta, J. J. Crochet, M. Chhowalla, S. Tretiak, M. A. Alam, H. L. Wang, and A. D. Mohite, Science 347, 522 (2015).

[9] I. W. Cho and M. Y. Ryu, Appl. Sci. Converg. Technol. 29, 28 (2020).

[10] M. Guo, P. Diao, and S. Cai, J. Solid State Chem. 178, 1864 (2005).

[11] J. Zhang and T. Pauporté, J. Phys. Chem. C 119, 14919 (2015).

[12] Y. Wen, Y. G. Tang, and G. Q. Yan, AIP Adv. 8, 095226 (2018).

[13] M. Nukunudompanich, G. Budiutama, K. Suzuki, K. Hasegawa, and M. Ihara, CrystEngComm 22, 2718 (2020). 